\title{
Postoperative interleukin-8 levels are related to the duration of coronary artery bypass grafting surgery and predict in-hospital postsurgical complications
}

\author{
Zsombor Mathe ${ }^{1 \#}$, Razvan Constantin Serban ${ }^{1 * \#}$, Irina Pintilie ${ }^{2}$, Cristina \\ Somkereki ${ }^{1}$, Adina Hutanu ${ }^{3}$, Alina Scridon ${ }^{3}$
}

\section{University of Medicine and Pharmacy of Tîrgu Mureș, Romania; Emergency Institute for Cardiovascular Diseases and Transplantation Tîrgu Mureș, Romania}

2. Emergency Institute for Cardiovascular Diseases and Transplantation Tîrgu Mureș, Romania; Center for Advanced Medical and Pharmaceutical Research Tîrgu Mureș, Romania

3. University of Medicine and Pharmacy of Tîrgu Mureș, Romania; Center for Advanced Medical and Pharmaceutical Research Tîrgu Mureș, Romania

\begin{abstract}
Introduction: The magnitude of the very early coronary artery bypass grafting (CABG)-related inflammatory response has been shown to influence post-CABG outcomes. However, the dynamics of the systemic inflammatory response to $C A B G$ beyond the very early postoperative phase and its relevance to clinical outcomes are not fully understood.

Methods: Circulating levels of several inflammatory markers were determined in 30 consecutive patients undergoing elective isolated on-pump CABG one day prior (D0-1), and 2 (D2) and 5 days post-CABG.

Results: $C A B G$ was associated with a significant increase in all studied inflammatory marker levels (all $p<0.05$ for D2 versus D0-1). D2 post-CABG IL-6 and IL-8 levels were both significantly positively correlated with extracorporeal circulation (ECC) and aortic clamping $(A C)$ times (all $p<0.05$ ), whereas a weaker correlation was observed between D2 post-CABG IL-8 levels and total surgery time $(r=0.42, p=0.02)$. In multiple regression analysis, D2 IL-8 levels independently predicted post-CABG kidney $(p=0.02)$ and liver $(p=0.04)$ dysfunction, as well as a sum of post-CABG major complications $\geq 2(p=0.04)$.

Conclusions: In this prospective study, longer duration of cardiopulmonary bypass caused a larger post-CABG inflammatory surge, whereas the duration of total CABG surgery had a less significant effect. IL-8 hyperresponders had greater risk of developing kidney and liver dysfunction and presented more major post-CABG complications. These data suggest that targeting the IL-8 pathway using antiinflammatory agents, or simply by shortening the duration of cardiopulmonary bypass could improve the in-hospital post-CABG outcomes in this population.
\end{abstract}

Keywords:coronary artery bypass grafting, in-hospital outcomes, inflammation, surgery times

Received: 22 $2^{\text {nd }}$ February 2018; Accepted: 20 th May 2018; Published: $13^{\text {th }}$ June 2018

\footnotetext{
* Corresponding author: Razvan Constantin Serban, Emergency Institute for Cardiovascular Diseases and Transplantation, Targu Mures, Romania .E-mail: serbanrazvan1@gmail.com

\# The first two authors contributed equally to the manuscript and both should be viewed as first author.
} 


\section{Introduction}

Coronary heart disease is one of the leading causes of death worldwide. The recent advances in percutaneous coronary interventions (PCI), the lower rates of complications, and the continuously increasing availability of this approach have considerably reduced the number of patients referred for coronary artery bypass grafting $(\mathrm{CABG})$ over the past decades (1). However, not all novel PCI-related approaches have met our expectations; bioresorbable scaffolds have been associated with very late stent thrombosis (2) and coronary aneurysm formation (3), whereas the safety of ticagrelor, one of the novel promising antiplatelet agents, and its benefit on mortality, stent thrombosis, and myocardial infarction have been significantly challenged (4). Furthermore, in patients with multivessel and left main disease, $\mathrm{CABG}$ continues to represent the gold standard (5).

Although post-CABG morbi-mortality has significantly declined over the past years, in-hospital mortality remains $>2 \%$ and complications continue to occur in up to one-third of patients (1). Many of these complications have been linked to patients' preoperative risk profile. However, the immediate postoperative course of this population is also dependent on intraoperative factors; extracorporeal circulation (ECC) and total surgery time have been linked to adverse in-hospital outcomes in previous studies $(6,7)$.

The very early inflammatory surge that follows CABG surgery has been incriminated in the occurrence of many post-CABG complications (8). Accumulating data indicate that higher magnitude of this very early CABG-related inflammatory response is associated with poorer short- and long-term outcomes (9), whereas administration of serine protease inhibitors has been associated with reduced post-CABG morbidity (10). However, it remains unclear how much of the inflammatory response that occurs in the very early post-CABG phase reflects the amount of trauma caused by the surgical procedure per se and how much of it is actually due to concomitant confounding factors such as the administration of protamine, of blood and/ or blood products, complement system activation by the contact of blood with the surface of the extracorporeal circuit and exposure of blood to abnormal shear forces, or to acute lesions due to electrical defibrillation. The dynamics of the systemic inflammatory response to $\mathrm{CABG}$ beyond the very early postoperative phase and its relevance to clinical outcomes are not fully understood.

Accordingly, we aimed to assess whether the previously reported impact of the duration of CABG on postoperative inflammatory marker levels is maintained beyond the very early post-CABG phase and to investigate whether the magnitude of this post-CABG inflammatory response contributes to patients' in-hospital postoperative outcomes.

\section{Material and methods}

\section{Study population}

Thirty consecutive patients treated by elective CABG in our center between March 2016 and June 2017 were studied prospectively. All patients were admitted to hospital for a firsttime elective $\mathrm{CABG}$ procedure, were $\geq 18$ years of age and were hemodynamically stable at the moment of inclusion. Patients were not included in the study if concomitant valve replacement or other intervention was planned to be performed within the same procedure, if they had systemic inflammatory diseases, or if they were on ongoing steroids or other anti-inflammatory drugs except for low doses of aspirin. All patients underwent surgery involving median sternotomy and standard ECC. Patients gave written informed consent; the research protocol complied with the Declaration of Helsinki and was approved by the local Ethics Committee. 
Age, gender, data regarding concomitant cardiovascular conditions (i.e., arterial hypertension, diabetes mellitus) and ongoing drug therapy were recorded for each patient. Left ventricular ejection fraction (LVEF) assessed by transthoracic echocardiography and the SYNergy between PCI with TAXUSTM and Cardiac Surgery (SYNTAX I) score were also recorded. For each patient, the duration of ECC, of aortic clamping, and the total duration of the CABG procedure were recorded.

\section{Inflammatory status assessment before and after coronary artery bypass grafting}

Venous blood samples were collected from each patient one day prior to CABG. Two additional venous blood samples were collected on days 2 and 5 after the procedure.

White blood cell (WBC) count was performed using the direct current detection method (Sysmex XP-300 Automated Hematology Analyzer; Sysmex Corporation, Japan). High-sensitivity C-reactive protein (hs-CRP) was determined by the immunoturbidimetric method (Automated Analyzer Cobas Integra 400; Roche Diagnostics), as described previously (11). Interleukins $1 \mathrm{~b}$ (IL-1b), 2 (IL-2), 6 (IL-6), and 8 (IL8) were quantified with bead-based Multiplex assays using the xMAP Luminex technology (Flexmap 3D analyzer; Luminex Corporation, Austin, TX).

\section{Post-coronary artery bypass grafting in-hospital outcomes}

Patients were prospectively monitored for clinically relevant post-CABG complications up to the time of discharge. Length of hospital stay was recorded. Prolonged hemodynamic instability, the occurrence of death, stroke, respiratory dysfunction, and in-hospital kidney and/ or liver dysfunction were recorded as major complications. Patients were considered to have prolonged hemodynamic instability if they required inotropic or vasopressor therapy for more than 24 hours after the procedure. Kidney dysfunction was defined as an increase in plasma creatinine $\geq 0.5 \mathrm{mg} / \mathrm{dL}$, whereas liver dysfunction was defined as a $\geq 3$-fold increase in liver transaminases. Other post-CABG complications, including surgical wound infection and post-CABG hemorrhage, were also noted.

\section{Statistical analysis}

Continuous variables are expressed as means \pm standard error of mean or, when skewed, median and interquartile range (IQR). Categorical data are presented using absolute numbers and percentages. The paired t-test or the Wilcoxon matched-pairs signed-ranks test were applied for comparison of inflammatory markers level at different time points. Spearman's rank correlation method or Pearson's correlation method were used to assess correlations between CABG-related parameters (i.e., durations of ECC, aortic clamping, and total CABG duration) and inflammatory markers levels, as well as between inflammatory markers levels and post-CABG in-hospital complications. Stepwise logistic regression analysis was used to assess the ability of post-CABG inflammatory markers levels to independently predict post-CABG in-hospital complications. The models were adjusted for age, history of arterial hypertension, and diabetes mellitus. All tests were two-sided and a $p$-value $<0.05$ was considered statistically significant. All data were computed using MedCalc for Windows, version 12.4.3.0 (MedCalc Software, Ostend, Belgium).

\section{Results}

\section{Baseline characteristics of the study popu- lation}

Out of the 30 patients included in this prospective study ( $80 \%$ male; mean age $60.1 \pm 9.6$ years), 29 patients $(96.7 \%)$ had a history of arte- 
rial hypertension and 11 patients $(36.7 \%)$ were diabetics. On admission, 27 patients (90.0\%) were receiving statins, $24(80.0 \%)$ were receiving angiotensin-converting enzyme inhibitors and/ or angiotensin II receptor blockers, 2 (6.7\%) were on antialdosteronics, and 27 (90.0\%) were on beta-blocker therapy. Median LVEF on admission was 50\% (IQR 45-55) and SYNTAX I score was $24.8 \pm 1.7$ points.

\section{Coronary artery bypass grafting surgery}

times and postoperative inflammatory sta-

tus

Overall, the total duration of CABG was $252.3 \pm 62.3 \mathrm{~min}$. Patients were in ECC for $104.5 \pm 38.8 \mathrm{~min}$, and aortic clamping time was $58.7 \pm 21.9 \mathrm{~min}$.

Inflammatory marker levels prior to $\mathrm{CABG}$, and 2 and 5 days after $C A B G$ are presented in
Figure 1. As expected, CABG was associated with a significant increase in all studied inflammatory marker levels. For most evaluated inflammatory markers, peak levels were recorded in postoperative day 2. Whereas plasma levels of IL-1b, IL-2, and IL-8 remained relatively constant (all $\mathrm{p}>0.05$ compared to postoperative day 2), WBC count, hs-CRP, and IL-6 levels significantly decreased by postoperative day 5 (all $\mathrm{p}<0.01$ compared to postoperative day 2). However, on postoperative day 5, WBC count, hs-CRP, and IL-6 levels continued to be significantly higher than prior to CABG surgery (all $\mathrm{p}<$ $0.0001)$. In addition, day 2 post-CABG hs-CRP (rho $=0.34, \mathrm{p}<0.0001)$, IL-6 (rho $=0.39, \mathrm{p}<$ $0.0001)$, and IL-8 (rho $=0.50, p<0.0001)$ levels significantly positively correlated with baseline, preoperative hs-CRP, IL-6, and IL-8 levels, respectively.

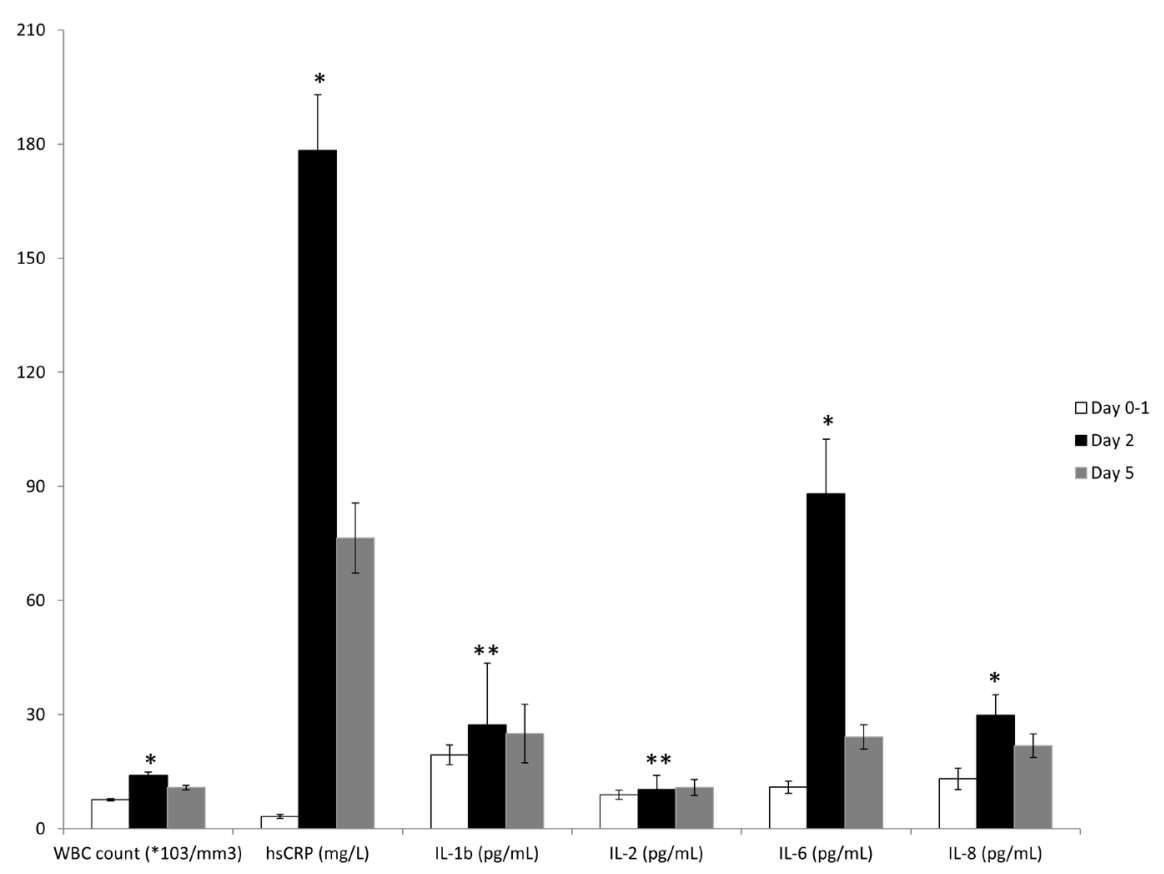

Fig. 1. Inflammatory marker levels prior to coronary artery bypass grafting (Day 0-1), and 2 (Day 2) and 5 (Day 5) days after coronary artery bypass grafting.

* - $\mathrm{p}<0.001$ versus Day $0-1 ; * *-\mathrm{p}<0.5$ versus Day $0-1$ using the Wilcoxon matched-pairs signed-ranks test; hsCRP - high-sensitivity C reactive protein; IL - interleukin; WBC - white blood cells

Data are expressed as means \pm standard error of the mean. 
There was no significant correlation between ECC, aortic clamping, or total surgery times and post-CABG WBC count, hs-CRP, IL-1b, or IL-2 levels (all $\mathrm{p}>0.05$ ). However, peak IL-8 levels (Figure 2) were significantly correlated with total surgery $($ rho $=0.42, \mathrm{p}=0.02), \mathrm{ECC}($ rho $=$

\section{Total surgery time}
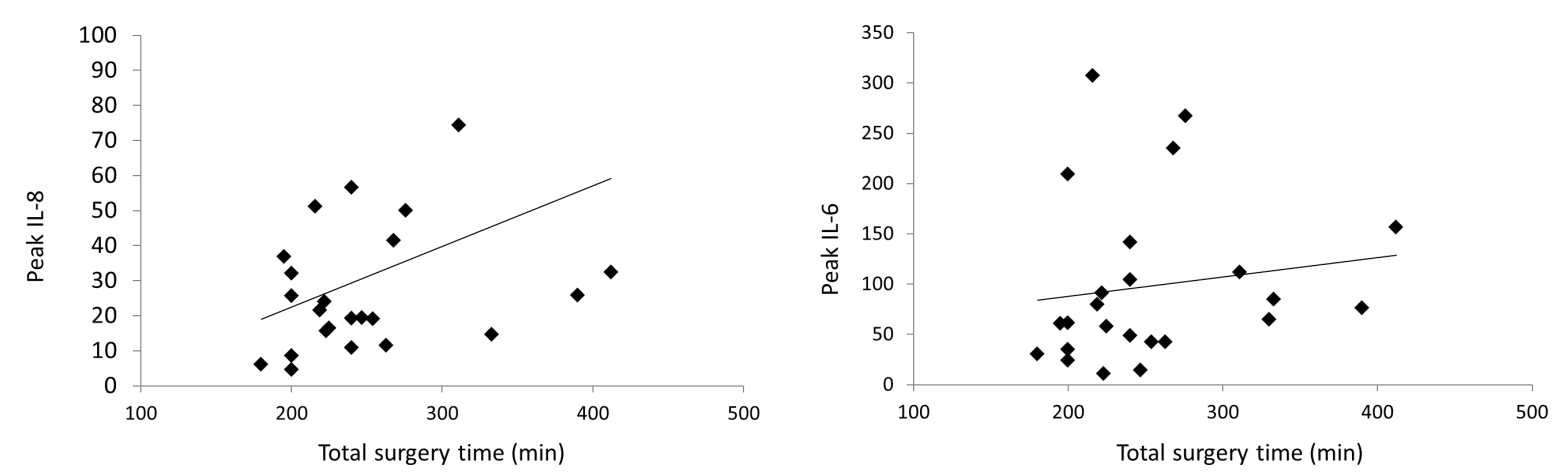

\section{Extracorporeal circulation time}
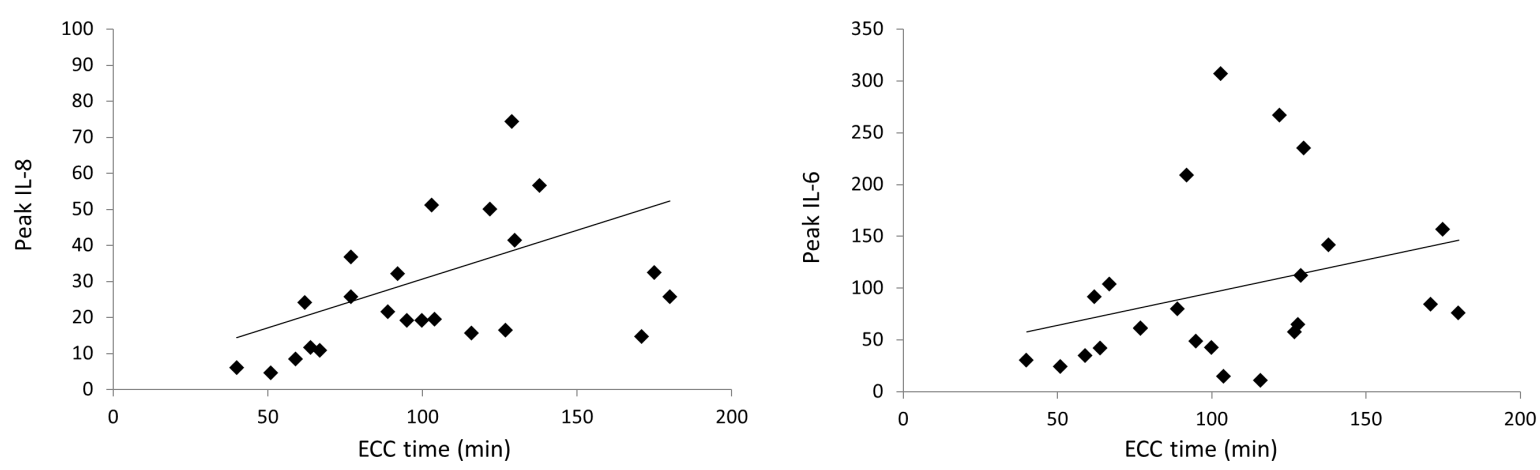

\section{Aortic clamping time}
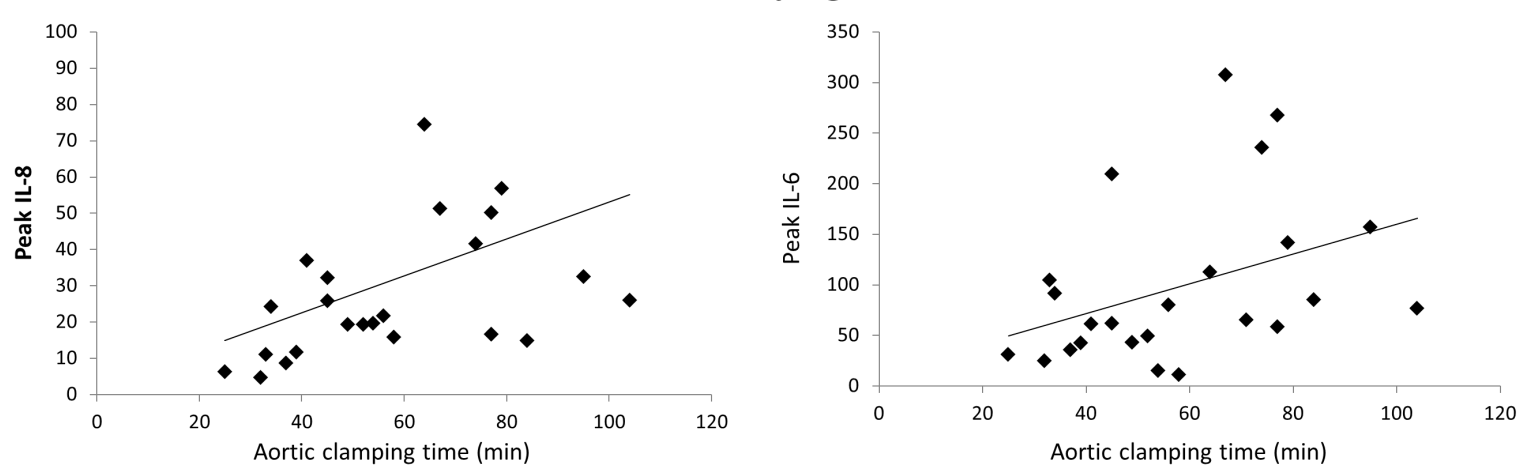

Figure 2. Relationship between absolute peak interleukin-8 and interleukin-6 levels and extracorporeal circulation, aortic clamping, and total surgery times. 
0.56, $\mathrm{p}<0.01$ ), and aortic clamping (rho $=0.57$, $\mathrm{p}<0.01)$ times. Meanwhile, peak IL-6 levels (Figure 2) were significantly correlated with ECC (rho $=0.40, p=0.03$ ) and aortic clamping (rho $=0.37, \mathrm{p}=0.04$ ) times, but not with total surgery time $(\mathrm{p}=0.09)$.

\section{Peak inflammatory marker levels and in-hospital post-coronary artery bypass grafting complications}

None of the study patients died during hospital stay and no patient required an intraaortic balloon pump during the perioperative period. Median length of hospital stay was 13 (IQR 11-18) days. Of the 30 patients included in the study, $22(73 \%)$ presented at least one post-CABG complication, and 8 patients $(26 \%)$ presented $>1$ complication. A detailed list of these complications is provided in Table 1.

There was no significant correlation between peak WBC count and any of the post-CABG complications (all p >0.05). Peak hs-CRP levels were significantly positively correlated with the length of hospital stay (rho $=0.42, \mathrm{p}=0.03$ ), whereas peak IL-1b, IL-2, and IL-6 levels significantly positively correlated with the duration of inotropic or vasopressor therapy (rho $=0.42$, $\mathrm{p}=0.03$; rho $=0.49, \mathrm{p}=0.02$; and rho $=0.45, \mathrm{p}$ $=0.02$, respectively) and with the occurrence of major complications (rho $=0.54, \mathrm{p}<0.01$; rho $=$ $0.52, \mathrm{p}=0.01$; and rho $=0.38, \mathrm{p}=0.04$, respectively). Peak IL-1b and IL-2 levels were also significantly positively correlated with the sum of major post-CABG complications ( $\mathrm{rho}=0.44$, $\mathrm{p}=0.02$ and rho $=0.53, \mathrm{p}<0.01$, respectively). Finally, peak IL-8 levels significantly positively correlated with the duration of inotropic or vasopressor therapy (rho $=0.42, p=0.03$ ), the occurrence of kidney (rho $=0.46, p=0.01)$ and liver (rho $=0.51, \mathrm{p}<0.01$ ) dysfunction, with the occurrence of major complications (rho $=0.39$, $p=0.03$ ), as well as with the sum of major postCABG complications ( $\mathrm{rho}=0.46, \mathrm{p}=0.01$ ).

In multiple regression analysis including age, history of arterial hypertension and diabetes mellitus, correlations between all other inflammatory markers and post-CABG complications were lost (all p >0.05), with the exception of peak IL-8 levels, which remained a strong independent predictor of post-CABG kidney ( $\mathrm{p}=$ $0.02)$ and liver $(p=0.04)$ dysfunction, as well as of a sum of post-CABG major complications $\geq 2$ $(\mathrm{p}=0.04)$.

\section{Discussions}

The main findings of the present study were that 1) CABG surgery was associated with

Table 1. In-hospital post-coronary artery bypass grafting complications

\begin{tabular}{lc}
\hline Complication & Complication rate \\
\hline Major complications & \\
Death & $0(0 \%)$ \\
Stroke & $1(3.3 \%)$ \\
Prolonged hemodynamic instability & $8(26.7 \%)$ \\
Kidney dysfunction & $9(30 \%)$ \\
Liver dysfunction & $5(16.7 \%)$ \\
$\quad$ Respiratory dysfunction & $5(16.7 \%)$ \\
\hline Other complications & \\
$\quad$ Surgical wound infection & $0(0 \%)$ \\
$\quad$ Post-CABG hemorrhage & $5(16.7 \%)$ \\
\hline
\end{tabular}

CABG - coronary artery bypass grafting

Data are expressed as absolute numbers and percentages. 
marked increase in WBC count, as well as in hs-CRP, IL-1b, IL-2, IL-6, and IL-8 levels; 2) longer duration of ECC and aortic clamping caused a larger post-CABG inflammatory surge, 3) whereas the duration of total $\mathrm{CABG}$ surgery had a less significant effect; and 4) peak IL-8 levels independently predicted post-CABG kidney and liver dysfunction, as well as a sum of post-CABG major complications $\geq 2$.

\section{Longer duration of extracorporeal circula- tion and aortic clamping leads to a larger postoperative inflammatory surge}

The pro-inflammatory effect of CABG is well-established and has been related, in addition to intrinsic myocardial trauma, to a multitude of factors including administration of protamine, of blood and/ or blood products, contact of the blood with the surface of the extracorporeal circuit and exposure of the blood to abnormal shear forces (12). Whereas these latter factors initiate inflammation during and immediately after CABG, protracted intrinsic myocardial sufferance is likely to become more relevant later on, after the very early post-CABG phase. Although the immediate inflammatory response has been related to a multitude of very early post-CABG complications, including prolonged hemodynamic instability, hyperthermia, or bleeding disorders, most post-CABG complications such as tissue edema, fever, arrhythmias, myocardial ischemia and dysfunction, respiratory distress syndrome, renal failure, or neurologic injury, generally occur days after the procedure (13). Meanwhile, experimental studies have demonstrated that complement system activation (13), but also IL-6 (14) and IL-8 (15) administration are followed by clinically relevant biological effects within hours and not days. Therefore, it seems reasonable to hypothesize that these relatively late complications are unlikely to be a consequence of the initial inflammatory response. Indeed, previous studies have demon- strated a biphasic pattern of tumor necrosis factor $\alpha$ (TNF $\alpha)$, IL-1b, IL-6, IL-8 (12,16), and complement activation (13), characterized by an early peak within the first hours after the end of cardiopulmonary bypass, return to near normal values by 24 hours, and then a second increase 48 hours after the procedure. Although the exact origin of this ensuing inflammatory reaction has not been established, its presence may be of greater discriminatory value for higher protracted myocardial injury $(17,18)$. The present study confirms that the levels of several inflammatory markers are elevated following $\mathrm{CABG}$, a finding that has been reported in varying degrees by a number of previous studies (9). Our results add to the already existing data and indicate IL- 8 as one of the most adequate markers for evaluating post-CABG inflammation, not only in the very early but also in the later postoperative phase.

Previous studies have demonstrated significant correlations between the duration of ECC and the magnitude of the very early inflammatory response $(9,19,20)$. This is not surprising, given that cardiopulmonary bypass is known to induce a systemic inflammatory response and that longer ECC duration increases the likelihood of blood and/ or blood product administration and prolongs the contact of the blood with the extracorporeal circuit and its exposure to abnormal shear forces, all of which have been associated with pro-inflammatory effects (21). However, the potential impact of ECC, aortic clamping, and/ or total surgery times on the inflammatory response beyond the very early postCABG phase has not been assessed previously. The present study indicates for the first time that longer duration of ECC and aortic clamping is associated with higher IL- 6 and IL- 8 levels 48 hours after $\mathrm{CABG}$ surgery, whereas the duration of total CABG surgery has a less significant or even negligible effect.

The significant correlation between ECC and aortic clamping time and post-CABG IL- 6 and 
particularly IL-8 levels, together with the weaker correlation between total surgery time and IL-8 and the lack of correlation between total surgery time and IL- 6 indicate cardiopulmonary bypass, and not surgical trauma itself as one of the major factors responsible for post-CABG inflammation and suggest that avoiding cardiopulmonary bypass may be associated with less inflammation and therefore with better postoperative outcomes. Indeed, previous studies have associated off-pump CABG with better long-term survival compared with on-pump CABG (22). However, others suggested that on-pump CABG may actually reduce in-hospital mortality, as well as late all-cause mortality compared to off-pump CABG $(23,24)$. Displacement of the heart to expose the target vessels during off-pump CABG, causing hemodynamic impairment, has been proposed to explain these findings (25). While the debate on the superiority of off-pump versus on-pump CABG still continues, it is obvious from the results of the present study that reduction of the post-CABG inflammatory surge by shortening the duration of cardiopulmonary bypass should be one of the goals of surgeons performing onpump CABG.

\section{Peak interleukin 8 levels independently predict post-coronary artery bypass com- plications}

A number of established cardiovascular risk factors are widely accepted predictors of postCABG complications. However, factors related to the surgical intervention itself, including the postoperative inflammatory surge, are expected to have a major impact on the postoperative course of these patients. Accordingly, after demonstrating the impact of ECC and aortic clamping time on day 2 post-CABG inflammatory markers levels, we thought to assess whether this larger inflammatory response is reflected into worse patients' clinical outcomes.
Our results show that IL- 6 and particularly IL-8 levels 48 hours after elective CABG surgery correlate with the duration of inotropic or vasopressor therapy, with the occurrence of kidney and liver dysfunction, as well as with the occurrence and the sum of major post-CABG complications. However, in multivariate analysis, neither peak IL-6 nor peak IL-8 levels remained independent predictors of the duration of inotropic or vasopressor therapy or of major post-CABG complications. However, "IL-8 hyperresponders" had a greater risk of developing kidney and liver dysfunction and presented more major post-CABG complications. Thus, the present study demonstrates that elevated day 2 post-CABG IL- 8 levels independently predict the later occurrence of a number of clinically relevant in-hospital complications after elective CABG procedures.

From a mechanistic point of view, these relatively late inflammatory marker levels may reflect more accurately the amount of intrinsic myocardial damage and may be less dependent on CABG-related confounding factors. Indeed, postoperative troponin $\mathrm{T}$ levels have been shown to correlate with early, mid-, and long-term morbi-mortality in CABG patients $(18,26-28)$, and troponin $\mathrm{T}$ levels at 48 hours after the $\mathrm{CABG}$ procedure have been associated with the highest prognostic power $(18,27,29)$. Similar results have been reported for creatine kinase-myocardial band (18). However, late assessment of inflammatory marker levels may be less useful for guiding early and intensified prophylactic strategies.

Meanwhile, our results and the results of previously published studies demonstrate that inflammation is clinically relevant following CABG and indicate IL- 8 as a potential target for reducing post-CABG complications. Glucocorticoids (30), interleukin 4 (31) and/ or IL-8 receptor antagonists may all prove useful in this regard. However, to date, only limited data are 
available regarding the potential benefit of IL-8 pathway inhibition in cardiovascular diseases. Alternatively, the present study suggests that benefit could be obtained by simply reducing the duration of ECC and aortic clamping.

Finally, the present study demonstrated that baseline hs-CRP, IL-6, and particularly IL-8 levels were significantly correlated with day 2 postoperative levels of these markers, suggesting that baseline IL-8 levels could be used to predict "IL-8 hyperresponders" and thus to identify the population at the highest risk of post-CABG exaggerated inflammatory response and complications, who would benefit the most from reduction of cardiopulmonary bypass duration and/ or other prophylactic strategies.

\section{Potential limitations}

Our study has a number of limitations. This is a single-center, observational study and therefore cannot ascribe causality. We evaluated a relatively small and homogenous population undergoing elective on-pump CABG surgery. Thus, although our results are in line with those of previous studies, these data cannot be generalized to the entire population of patients undergoing CABG. Also, we performed a limited range of inflammatory marker measurements (preoperative, and 2 and 5 days postoperative); inflammatory marker levels during and immediately after $\mathrm{CABG}$ were not determined. Thus, the presence of a second inflammatory peak in postoperative day 2 was not documented in the present study and we cannot exclude the fact that inflammatory marker levels measured 48 hours after $\mathrm{CABG}$ reflect the natural descending progression of the initial inflammatory surge. However, previous studies have repeatedly demonstrated the presence of a second inflammatory peak, at least for TNF $\alpha$, IL-1b, IL-6, IL8 , and the complement system. Above all, the present study should be viewed as a basis for future larger-scale studies.

\section{Conclusions}

This prospective study demonstrated that elective $C A B G$ surgery is associated with a significant inflammatory response. Longer duration of ECC and aortic clamping caused a larger increase in day 2 post-CABG IL- 6 and IL- 8 levels, whereas the duration of total CABG surgery had a less significant (for IL-8) or even negligible (for IL-6) effect. At their turn, IL-8 levels measured 48 hours after CABG independently predicted postoperative kidney and liver dysfunction, as well as a sum of major post-CABG complications $\geq 2$. Taken together, these data suggest that targeting the IL-8 pathway using antiinflammatory agents, or simply by shortening the duration of ECC and aortic clamping, could improve the in-hospital post-CABG outcomes in this population.

\section{Acknowledgments}

This study was funded by the University of Medicine and Pharmacy of Tîrgu Mureș (research grant number 17800/1/22.12.2015).

\section{Conflicts of interest}

The authors report no conflicts of interest.

\section{Authors' contribution}

$\mathrm{ZM}$, CS, and AH contributed to acquisition of data, critical revision of the manuscript for important intellectual content, and final approval of the version to be published. RCS and AS contributed to conception and design, analysis and interpretation of data, drafting of the manuscript, and final approval of the version to be published. IP contributed to acquisition and interpretation of data, critical revision of the manuscript for important intellectual content, and final approval of the version to be published. 


\author{
Abbreviations \\ AC - aortic clamping \\ $\mathrm{CABG}$ - coronary artery bypass grafting \\ D0-1 - the day before the day of surgery \\ D2 - second day after the surgery \\ ECC - extracorporeal circulation \\ hs-CRP - high-sensitivity C-reactive protein \\ IL- $1 \mathrm{~b}$ - interleukin $1 \mathrm{~b}$ \\ IL-2 - interleukin 2 \\ IL-6 - interleukin 6 \\ IL-8 - interleukin 8 \\ IQR - interquartile range \\ LVEF - left ventricular ejection fraction \\ PCI - percutaneous coronary intervention \\ SYNTAX I - SYNergy between PCI with TAX- \\ US $^{\text {TM }}$ and Cardiac Surgery \\ TNF $\alpha$ - tumor necrosis factor \\ WBC - white blood cells
}

\section{References}

1. Moazzami K, Dolmatova E, Maher J, Gerula C, Sambol J, Klapholz M, et al. In-hospital outcomes and complications of coronary artery bypass grafting in the United States between 2008 and 2012. J Cardiothorac Vasc Anesth. 2017;31(1):19-25. DOI: 10.1053/j. jvca.2016.08.008

2. Yamaji K, Ueki Y, Souteyrand G, Daemen J, Wiebe $\mathrm{J}$, Nef $\mathrm{H}$, et al. Mechanisms of very late bioresorbable scaffold thrombosis: The INVEST registry. J Am Coll Cardiol. 2017;70(19):2330-44. DOI: 10.1016/j. jacc.2017.09.014

3. Șerban R, Scridon A, Dobreanu D, Elkaholut A. Coronary artery aneurysm formation within everolimus-eluting bioresorbable stent. Int J Cardiol. 2014;177:e4-5. DOI: 10.1016/j.ijcard.2014.07.197

4. DiNicolantonio JJ, D'Ascenzo F, Tomek A, Chatterjee S, Niazi AK, Biondi-Zoccai G. Clopidogrel is safer than ticagrelor in regard to bleeds: a closer look at the PLATO trial. Int J Cardiol. 2013;168(3):1739-44. DOI: 10.1016/j.ijcard.2013.06.135

5. Mohr FW, Morice MC, Kappetein AP, Feldman TE, Ståhle E, Colombo A, et al. Coronary artery bypass graft surgery versus percutaneous coronary intervention in patients with three-vessel disease and left main coronary disease: 5-year follow-up of the randomised, clinical SYNTAX trial. Lancet. 2013;381:629-38. DOI: 10.1016/S0140-6736(13)60141-5

6. Piątek J, Kędziora A, Konstanty-Kalandyk J, Kiełbasa G, Olszewska M, Song BH, et al. Risk factors for in-hospital mortality after coronary artery bypass grafting in patients 80 years old or older: a retrospective case-series study. PeerJ. 2016;4:e2667. DOI: 10.7717/ peerj. 2667

7. Turrentine FE, Wang H, Simpson VB, Jones RS. Surgical risk factors, morbidity, and mortality in elderly patients. J Am Coll Surg. 2006;203(6):865-77. DOI: 10.1016/j.jamcollsurg.2006.08.026

8. Poole L, Kidd T, Leigh E, Ronaldson A, Jahangiri M, Steptoea A. Depression, C-reactive protein and length of post-operative hospital stay in coronary artery bypass graft surgery patients. Brain Behav Immun. 2014;37(100):115-21. DOI: 10.1016/j.bbi.2013.11.008

9. Holmes JH 4th, Connolly NC, Paull DL, Hill ME, Guyton SW, Ziegler SF, et al. Magnitude of the inflammatory response to cardiopulmonary bypass and its relation to adverse clinical outcomes. Inflamm Res. 2002;51(12):579-86. DOI: 10.1007/PL00012432

10. Royston D. Serine protease inhibition prevents both cellular and humoral responses to cardiopulmonary bypass. J Cardiovasc Pharmacol. 1996;27(Suppl. 1):S429. DOI: 10.1097/00005344-199600001-00010

11. Cernea S, Şular FL, Huţanu A, Voidăzan S. Markers of cognitive impairment in patients with type 2 diabetes. Rev Rom Med Lab. 2016;24(2):161-76. DOI: 10.1515/ rrlm-2016-0023

12. Hennein HA, Ebba H, Rodriguez JL, Merrick SH, Keith FM, Bronstein MH, et al. Relationship of the proinflammatory cytokines to myocardial ischemia and dysfunction after uncomplicated coronary revascularization. J Thorac Cardiovasc Surg. 1994;108(4):62635.

13. Bruins P, te Velthuis H, Yazdanbakhsh AP, Jansen PG, van Hardevelt FW, de Beaumont EM, et al. Activation of the complement system during and after cardiopulmonary bypass surgery: postsurgery activation involves C-reactive protein and is associated with postoperative arrhythmia. Circulation. 1997;96(10):3542-8. DOI: 10.1161/01.CIR.96.10.3542

14. Finkel MS, Oddis CV, Jacob TD, Watkins SC, Hattier BG, Simmons RL. Negative inotropic effects of cytokines on the heart mediated by nitric oxide. Science. 
1992;257:387-9. DOI: 10.1126/science. 1631560

15. Van Zee KJ, Fischer E, Hawes AS, Hébert CA, Terrell TG, Baker JB, et al. Effects of intravenous IL-8 administration in nonhuman primates. J Immunol. 1992;148(6):1746-52.

16. Mitchell JD, Grocott HP, Phillips-Bute B, Mathew JP, Newman MF, Bar-Yosef S. Cytokine secretion after cardiac surgery and its relationship to postoperative fever. Cytokine. 2007;38(1):37-42. DOI: 10.1016/j. cyto.2007.04.009

17. Metinko A, Kunkel S, Standiford T, Streter R. Monocyte expression of interleukin-8 in response to oxidant stress. FASEB J Abstracts Part 1. 1991:1941;A704 (Abstract).

18. Søraas CL, Friis C, Engebretsen KV, Sandvik L, Kjeldsen SE, Tønnessen T. Troponin $\mathrm{T}$ is a better predictor than creatine kinase-MB of long-term mortality after coronary artery bypass graft surgery. Am Heart J. 2012;164(5):779-85. DOI: 10.1016/j.ahj.2012.05.027

19. Kawamura T, Wakusawa R, Okada K, Inada S. Elevation of cytokines during open heart surgery with cardiopulmonary bypass: participation of interleukin 8 and 6 in reperfusion injury. Can J Anaesth. 1993;40(11):101621. DOI: 10.1007/BF03009470

20. Wu ZK, Laurikka J, Vikman S, Nieminen R, Moilanen E, Tarkka MR. High postoperative interleukin-8 levels related to atrial fibrillation in patients undergoing coronary artery bypass surgery. World J Surg. 2008;32(12):2643-9. DOI: 10.1007/s00268-008-97587

21. Kirklin, JW, Barratt-Boyes, BG. Hypothermia, circulatory arrest, and cardiopulmonary bypass. In: Cardiac surgery. 2nd ed. Churchill Livingstone, New York; 1993: 61-128.

22. Gurbuz O, Kumtepe G, Ercan A, Yolgosteren A, Ozkan H, Karal IH, et al. A comparison of off- and onpump beating-heart coronary artery bypass surgery on long-term cardiovascular events. Cardiovasc J Afr. 2017;28(1):30-5. DOI: 10.5830/CVJA-2016-049

23. Wang J, Yu W, Zhao D, Liu N, Yu Y. In-hospital and long-term mortality in 35,173 Chinese patients undergoing coronary artery bypass grafting in Beijing: impact of sex, age, myocardial infarction, and car- diopulmonary bypass. J Cardiothorac Vasc Anesth. 2017;31(1):26-31. DOI: 10.1053/j.jvca.2016.08.004

24. Takagi H, Umemoto T. All-Literature Investigation of Cardiovascular Evidence (ALICE) Group: Worse long-term survival after off-pump than on-pump coronary artery bypass grafting. J Thorac Cardiovasc Surg. 2014;148:1820-9. DOI: 10.1016/j.jtcvs.2014.05.034

25. Mathison M, Edgerton JR, Horswell JL, Akin JJ, Mack MJ. Analysis of hemodynamic changes during beating heart surgical procedures. Ann Thorac Surg. 2000;70:1355-60. DOI: 10.1016/S00034975(00)01590-3

26. Göber V, Hohl A, Gahl B, Dick F, Eigenmann V, Carrel TP, et al. Early troponin $\mathrm{T}$ and prediction of potentially correctable in-hospital complications after coronary artery bypass grafting surgery. PLoS One. 2013;8(9):e74241. DOI: 10.1371/journal. pone. 0074241

27. Lehrke S, Steen H, Sievers HH, Peters H, Opitz A, Müller-Bardorff $\mathrm{M}$, et al. Cardiac troponin $\mathrm{T}$ for prediction of short- and long-term morbidity and mortality after elective open heart surgery. Clin Chem. 2004;50(9):1560-7. DOI: 10.1373/ clinchem.2004.031468

28. Kathiresan S, Servoss SJ, Newell JB, Trani D, MacGillivray TE, Lewandrowski K, et al. Cardiac tropo$\operatorname{nin} \mathrm{T}$ elevation after coronary artery bypass grafting is associated with increased one-year mortality. Am J Cardiol. 2004;94(7):879-81. DOI: 10.1016/j.amjcard.2004.06.022

29. Carrier M, Pellerin M, Perrault LP, Solymoss BC, Pelletier LC. Troponin levels in patients with myocardial infarction after coronary artery bypass grafting. Ann Thorac Surg. 2000;69(2):435-40. DOI: 10.1016/S00034975(99)01294-1

30. Mukaida N, Shiroo M, Matsusima K. Genomic structure of the human monocyte-derived neutrophil chemotactic factor IL-8. J Immunol. 1989;143:1366-71.

31. Standiford TJ, Strieter RM, Chensue SI, Westwick J, Kasahara K, Kunkel SL. IL-4 inhibits the expression of IL-8 from stimulated monocytes. J Immunol. 1990; $145: 1435-9$. 